HD-THEP-92-39

Preprint TU-411

September, 1992

\title{
Quantum Deformation of BRST Algebra ${ }^{\ddagger}$
}

\author{
Satoshi Watamura \\ Department of Physics, College of General Education, \\ Tohoku University, Kawauchi, \\ Aobaku, Sendai 980, Japan. \\ bitnet:watamura@jpntuvm0 \\ Institute für Theoretische Physik \\ Universität Heidelberg \\ Philosophenweg 16, D-6900
}

\begin{abstract}
We investigate the $q$-deformation of the BRST algebra, the algebra of the ghost, matter and gauge fields on one spacetime point using the result of the bicovariant differential calculus. There are two nilpotent operations in the algebra, the BRST transformation $\delta_{B}$ and the derivative $d$. We show that one can define the covariant commutation relations among the fields and their derivatives consistently with these two operation as well as the *-operation, the antimultiplicative inner involution.
\end{abstract}

\footnotetext{
${ }^{\ddagger}$ This work is partly supported by Alexsander von Humboldt Foundation.
} 


\section{Introduction}

It is an interesting question whether one can construct a $q$-analogue of the gauge theory by taking the quantum group [Dri,FRT,Jim,Wor1] as a symmetry. One of the interesting possibilities of such a $q$-deformed theory is that the deformation parameter $q$ may play a role of a regularization parameter. Furthermore, since the quantum group is provided by a noncommutative algebra, in such a theory the noncommutative geometry [Connes] plays a basic role like the differential geometry in the usual gauge theory.

There are some proposals to this problem [Are,Ber,BM,Hira,IP,WuZ]. However, it seems that there are still conceptual problems concerning the definition of the gauge transformation when we take the quantum group as an algebraic object of the gauge symmetry. Since the quantum group is formulated in the language of the Hopf algebra, it forces us to formulate the whole theory in algebraic language. Thus the gauge transformation will be represented in an abstract language and the term of the transformation parameter becomes obscure. Even when we consider only the infinitesimal transformation, we have still the question of the definition of the infinitesimal parameters.

One of the alternative formulations of the gauge theory is given by the BRST formalism [BRST,KO]. There, the gauge transformation parameter is replaced by the ghost fields and becomes an object of equal level with the matter and the gauge fields. Therefore, when we consider the $q$-deformation of the gauge theory, it is very natural to consider the $q$-deformed field algebra starting with the BRST formalism.

In this paper we construct a $q$-deformation of the BRST algebra which is the algebra of the gauge fields, the ghost fields and appropriate matter fields on one spacetime point. The gauge transformation of the theory is replaced by the BRST transformation which is represented by a nilpotent "differential operator" $\delta_{B}$.

The paper is organized as follows. In section 1, we give a collection of some results of the bicovariant differential calculus on the quantum group which we need for the later investigation. In section 2 , we discuss about the algebraic properties of the gauge transformation and we give the general conditions that the $q$-deformed BRST algebra must satisfy. Following this general framework, we define the algebra and prove various consistencies in section 3. Section 4 is devoted to discussions.

For the notation concerning the Hopf algebra [Abe], we take: the coproduct $\Delta$, the antipode $\kappa$ and the counit $\varepsilon$ (see also ref.[CW] for our notation). Through this paper the upper case roman index $I, J, K, L$ runs $0,-, 3,+$ and the lower case index like $a, b, c, d$ runs over the label of the adjoint representation, $-, 3,+$, otherwise we specify explicitly.

\section{Bicovariant Differential Calculus}

Before we start to construct the BRST algebra, let us briefly recall some results of the bicovariant differential calculus [Wor2,Rosso,Jur,CSWW]. The one-forms are defined by the right invariant bases $\theta_{j}^{i}(i, j=1,2)$ where $\theta_{j}^{i *}=\theta_{i}^{j}$. Using the spinor metric

$$
\epsilon^{k l}=\left(\begin{array}{cc}
0 & -q^{-\frac{1}{2}} \\
q^{\frac{1}{2}} & 0
\end{array}\right)
$$


we define $\theta^{i j}=\theta_{k}^{i} \epsilon^{k j}$, then they have the commutation relation

$$
a \theta^{i j}=\theta^{k l}\left(a * \mathbf{L}_{k l}^{i j}\right)
$$

for $\forall a \in F u n_{q}(S U(2))$.

$\mathbf{L}$ is the functional $\operatorname{Fun}_{q}(S U(2)) \rightarrow \mathrm{C}$ defined by

$$
\mathbf{L}_{k l}^{i j}=\left(L_{-l}^{j} * L_{+k}^{i}\right) \circ \kappa
$$

where the functionals $L_{ \pm}$and the convolution product are defined in the appendix A. [The functionals $f_{ \pm}$appearing in ref.[CSWW] equivalent to the $L_{ \pm}$in ref.[FRT] which we use here. Thus, $\mathbf{L}_{k l}^{i j}$ is equivalent to the functional $f_{\mathrm{Ad} k l}^{i j} \circ \kappa$ in ref.[CSWW].]

The right invariant bases $\theta^{i j}$ can be split into two part as a left comodule : the adjoint representation $\theta^{a}(a=-, 3,+)$ and the singlet $\theta^{0}$. We use the $q$-Pauli matrices $\sigma_{i j}^{I}$ and $\sigma_{I}^{i j}$ where $\sigma_{i j}^{I} \sigma_{J}^{i j}=\delta_{J}^{I}$ $(I=0,-, 3,+)$ and

$$
\sigma_{k l}^{0}=\frac{q}{\sqrt{Q}} \epsilon_{k l}, \quad \sigma_{k l}^{+}=\left(\begin{array}{cc}
0 & 0 \\
0 & -1
\end{array}\right), \quad \sigma_{k l}^{-}=\left(\begin{array}{ll}
1 & 0 \\
0 & 0
\end{array}\right), \quad \sigma_{k l}^{3}=\frac{-1}{\sqrt{Q}}\left(\begin{array}{cc}
0 & q^{\frac{1}{2}} \\
q^{-\frac{1}{2}} & 0
\end{array}\right),
$$

where $Q=q+q^{-1}$ and $\epsilon_{k l}=-\epsilon^{k l}$. With these $q$-Pauli matrices we can write the projectors as

$$
\begin{aligned}
& \mathcal{P}_{A k l}^{i j}=\sigma_{0}^{i j} \sigma_{k l}^{0}, \\
& \mathcal{P}_{S k l}^{i j}=\sigma_{a}^{i j} \sigma_{k l}^{a},
\end{aligned}
$$

where $\mathcal{P}_{S}\left(\mathcal{P}_{A}\right)$ is the projector for the $q$-(anti)symmetric product. Using these $q$-Pauli matrices we define

$$
\theta^{I} \equiv \sigma_{i j}^{I} \theta^{i j}
$$

The $q$-deformed exterior derivative $\mathbf{d}$ is defined as a map from $F u n_{q}(G)$ to the bimodule defined with the basis $\theta$ requiring that the nilpotency and the Leibniz rule hold in the standard way [Wor2]. Such an operation can be defined simply as the commutator with the singlet component $\theta^{0}$ as [CSWW]

$$
\mathbf{d} a=\frac{i g}{\omega}\left[\theta^{0}, a\right]_{-}
$$

for any element $a \in \operatorname{Fun}_{q}(S U(2))$, where $\omega=q-q^{-1}, i=\sqrt{-1}$ and $g$ is a non-zero real constant. [The relation of the constant $g$ with the constant $N_{0}$ in ref.[CSWW] is $g=\frac{-i \sqrt{Q}}{q N_{0}}$.] Since $\mathbf{d} a$ is an element of the bicovariant bimodule, we can expand it with the basis as

$$
\mathbf{d} a=\theta^{I}\left(a * \chi_{I}\right) \quad,
$$

where the right invariant vector field $\chi_{i j}$ is given by

$$
\chi_{I} \equiv \sigma_{I}^{i j} \chi_{i j}=\sigma_{I}^{i j} \frac{i g}{\omega}\left(\sigma_{i j}^{0} \varepsilon-\sigma_{k l}^{0} \mathbf{L}_{i j}^{k l}\right) .
$$

We can consider the functional $\chi_{I}$ as a differential operator. The Leibniz rule is given by the coproduct of $\chi_{I}$ :

$$
\begin{aligned}
\left(a b * \chi_{I}\right) & =(a \otimes b) * \Delta\left(\chi_{I}\right) \\
& =\left(a * \chi_{I}\right) b+\left(a * \mathbf{L}_{I}^{J}\right)\left(b * \chi_{J}\right)
\end{aligned}
$$


One of the suggesting relation given by the bicovariant differential calculus is the $q$-analogue of the Maurer-Cartan equation. In ref.[CSWW], we gave the expression in a more familiar form:

$$
\begin{aligned}
& \mathbf{d} \theta^{0}=0, \\
& \mathbf{d} \theta^{a}=\frac{-i g}{q^{2}+q^{-2}} f_{b c}^{a} \theta^{b} \wedge \theta^{c},
\end{aligned}
$$

where $\wedge$ is the $q$-deformed exterior product. The $f_{b c}^{a}$ is the $q$-analogue of the structure constants. Using the general formula for the structure constants in ref.[CSWW] (See also ref.[Carow]), we obtain them for the $\operatorname{Fun}_{q}(S U(2))$ as

$$
\begin{aligned}
& f_{+3}^{+}=q \quad f_{3+}^{+}=-q^{-1}, \\
& f_{3-}^{-}=q \quad f_{-3}^{-}=-q^{-1}, \\
& f_{-+}^{3}=1 \quad f_{+-}^{3}=-1 \quad f_{33}^{3}=q-q^{-1} .
\end{aligned}
$$

The Maurer-Cartan equation and the definition of the $\chi_{I}$ given in eq.(1.10), we can deduce the commutation relation among the $q$-vector fields $\chi_{I}$. We find that $\chi_{0}$ is central and actually proportional to the second Casimir operator [Weich,CSWW]. The commutation relations of others are given by

$$
\mathbf{P}_{A d b c}^{b^{\prime} c^{\prime}}\left(\chi_{b^{\prime}} * \chi_{c^{\prime}}\right)=\frac{-1}{q^{2}+q^{-2}} f_{b c}^{a} \mathbf{p} * \chi_{a}
$$

where the functional $\mathbf{p}$ is central and

$$
\mathbf{p}=i g \varepsilon-\omega \chi_{0}
$$

(See also eq.(5.50) in ref.[CW].) The matrix $\mathbf{P}_{A d}=\left(\mathcal{P}_{S}, \mathcal{P}_{A}\right)+\left(\mathcal{P}_{A}, \mathcal{P}_{S}\right)$ is an operator projecting the tensor onto the $q$-antisymmetric part, where $\left(\mathcal{P}_{r}, \mathcal{P}_{r^{\prime}}\right)$ is defined in eq.(7.4) of ref.[CSWW] (see appendix B). $\mathbf{P}_{A d c d}^{a b}$ is the projector restricted to the product of two adjoint representations. Thus l.h.s. of eq.(1.15) gives the $q$-analogue of the usual commutator of the generators.

For the $\operatorname{Fun}_{q}(S U(2))$ calculus we can write

$$
\mathbf{P}_{A d c d}^{a b}=\frac{-1}{q^{2}+q^{-2}} f_{c d}^{a^{\prime}} f_{a^{\prime}}^{a b}
$$

where $f_{a}^{b c} \equiv-f_{b c}^{a}$. Therefore, it is straightforward to evaluate these projection operators and using that result, eq.(1.15) is written as

$$
\begin{aligned}
q^{-1} \chi_{3} * \chi_{+}-q \chi_{+} * \chi_{3} & =\mathbf{p} * \chi_{+}, \\
q \chi_{3} * \chi_{-}-q^{-1} \chi_{-} * \chi_{3} & =-\mathbf{p} * \chi_{-}, \\
\chi_{+} * \chi_{-}-\chi_{-} * \chi_{+}-\omega \chi_{3} * \chi_{3} & =\mathbf{p} * \chi_{3}
\end{aligned}
$$

In the limit $q \rightarrow 1$, the operator $\mathbf{p}$ is proportional to the counit and we get the standard commutation relation of generators of $S U(2)$. 


\section{Gauge Transformation and BRST Formalism}

\subsection{Gauge Transformation}

As we explained in the introduction, we need to represent the gauge theory using an appropriate algebra languages which fits to the Hopf algebra structure. Thus, let us first reconsider the gauge and the BRST transformation in the non-deformed theory. We take $S U(2)$ gauge theory as an example but the result applies to the general group.

When we consider the usual non-deformed gauge theory with a symmetry group $S U(2)$, the matter like a lepton is represented by the field which is the section of the associated fiber bundle of the structure group $S U(2)$ with the spacetime as a base manifold $B$. Thus the algebra of the matter fields is the algebra of all possible sections.

Under the gauge transformation, the matter field $\Psi$ is transformed according to its representation. Giving the $S U(2)$ valued function $g(x) \in S U(2)$ on the base manifold $B \ni x$, when the matter is of the fundamental representation the gauge transformation of the matter $\Psi^{i}(x)$ can be written as

$$
\left[\Psi^{i}(x)\right]^{g}=M_{j}^{i}(g(x)) \Psi^{j}(x),
$$

where $(i, j=1,2)$. We wrote the gauge transformation matrix as $M_{j}^{i}(g(x))$ to clarify the algebraic structure. The matrix element $M_{j}^{i}$ maps the $g(x)$ to the complex valued function on the base manifold and thus pointwise $M_{j}^{i}$ is an element of the $F u n(S U(2))$. Therefore, the gauge transformation property of the matter field can be translated into the algebraic language such that the algebra of matter fields is the (left)comodule algebra, and there is a pointwise (left)coaction $\Delta_{L}$ of $\operatorname{Fun}(S U(2))$ on the field $\Psi$ :

$$
\Delta_{L}(\Psi)=\sum_{s} T_{s} \otimes \Psi^{s}
$$

where $T_{s} \in \operatorname{Fun}(S U(2))$ are matrix elements of the representation corresponding to the matter $\Psi$. For the fundamental representation eq.(2.2) is $\Delta_{L}\left(\Psi^{i}\right)=M_{j}^{i} \otimes \Psi^{j}$ and with the corresponding argument we get eq.(2.1).

The infinitesimal transformation corresponding to the transformation (2.1) can be written as

$$
\delta_{\xi}\left(\Psi^{i}(x)\right)=\xi^{a}(x) \chi_{a}\left(M_{j}^{i}\right) \Psi^{j}(x)
$$

where $a=-, 3,+$ is the label of the adjoint representation of $S U(2), \xi^{a}$ is the gauge parameter which is the real function of the spacetime and $\chi_{a}\left(M_{j}^{i}\right)$ is a $2 \times 2$ matrix. In the non-deformed case we can identify $\chi_{a}$ with the right invariant vector fields which are considered as the linear functionals $\operatorname{Fun}(S U(2)) \rightarrow \mathrm{C}$ with the evaluation

$$
\chi_{a}\left(M_{j}^{i}\right)=\left.L_{a}^{\mu} \frac{\partial}{\partial \phi^{\mu}} M_{j}^{i}\left(g\left(\phi^{\nu}\right)\right)\right|_{\text {at unity }},
$$

where $g\left(\phi^{\mu}\right)$ is the group element parametrized by $\phi^{\mu}$ and $L_{a}^{\mu}$ is the component of the right invariant vector field. The r.h.s. gives the Pauli matrix for the $S U(2)$ case and thus eq. 2.3) is the familiar infinitesimal transformation. The above structure can be translated into the algebraic language as follows:

The infinitesimal transformation $\delta_{\xi}$ of the matter field $\Psi$ can be represented by the vector fields $\chi_{a}$ and the infinitesimal parameter $\xi^{a}$ as

$$
\delta_{\xi} \Psi=\xi^{a}\left(\Psi * \chi_{a}\right)
$$


where $(\cdot * \cdot)$ denotes the convolution product of a comodule with a functional. For detail see appendix A. For the fundamental representation, using the definition (A.10) it is easy to show that the formula (2.5) is equivalent to eq.(2.3).

Using the above algebraic representations, we may consider the $q$-analogue of the finite and the infinitesimal gauge transformation which we will discuss elsewhere. Here we want to concentrate on the $q$-deformation of the BRST algebra which seems the most appropriate algebra to consider the $q$-deformation of the gauge theory.

The BRST transformation of the matter field is defined by replacing the gauge parameter $\xi^{a}$ by the ghost field $C^{a}[\mathrm{FP}]$. Thus the BRST transformation can be written as

$$
\delta_{B} \Psi=C^{a}\left(\Psi * \chi_{a}\right)
$$

For the fundamental representation this is

$$
\delta_{B} \Psi^{i}=C^{a} \chi_{a}\left(M_{j}^{i}\right) \Psi^{j}
$$

Replacing the $\chi_{a}\left(M_{j}^{i}\right)$ with the Pauli matrix this is a familiar BRST transformation.

\subsection{Definition of $q$-deformed BRST Algebra}

After the above preparation we specify the properties of the $q$-deformed BRST algebra which we will construct in the next section. We extract appropriate properties from the non-deformed BRST formalism and impose them as the condition which our algebra should satisfy. We also require that in general in the limit $q \rightarrow 1$, we always get the algebra equivalent to the non-deformed one.

The BRST algebra is the algebra which contains the matter fields $\Psi$ and the gauge fields $A^{I}$ and the ghosts $C^{I}$ which are the standard field contents of the BRST formalism. The suffix $I$ corresponds to the adjoint representation in non-deformed case. However in the $q$-deformed case we only require that it contains the adjoint representation and allow to add a singlet component like the right invariant basis $\theta_{j}^{i}$ in the bicovariant differential calculus.

In the field theory, we have the spacetime derivative $d$ and therefore, we also require the existence of the map $d$ in the algebra which maps:

$$
\left(\Psi, A^{I}, C^{I}\right) \stackrel{d}{\longrightarrow}\left(d \Psi, d A^{I}, d C^{I}\right) \stackrel{d}{\longrightarrow} 0 .
$$

To construct the algebra we treat the fields $d \Psi, d A^{I}$ and $d C^{I}$ as independent generators from the original fields and then require the consistency with the above map $d$.

\section{Definition 1:}

The BRST algebra $\mathcal{A}_{B}$ is a comodule algebra over $\operatorname{Fun}_{q}(G)$ which is generated by the following set of the comodules:

$$
\mathcal{A}_{B}=\mathrm{C}<C^{I}, \Psi, A^{I}, d C^{I}, d \Psi, d A^{I}>/ \mathcal{I}
$$

where $C^{I}$ represents the ghost, $\Psi$ the matter and $A^{I}$ the gauge fields. $\mathcal{I}$ is a set of the covariant commutation relations among these comodules, which we shall determine in the next section.

In the non-deformed BRST formalism of the gauge theory there are two nilpotent operations, the exterior derivative $d$ and the BRST transformation $\delta_{B}$. We also require the corresponding structure in the algebra and that they keep the following properties in the $q$-deformed case: 


\section{Condition A :}

The BRST algebra possesses the following operations:

1. There exists an operation $\delta_{B}$ in the algebra $\mathcal{A}_{B}$ such that

(a) the $\delta_{B}$ operation satisfies the Leibniz rule in the graded sense,

(b) $\delta_{B}^{2}=0$.

2. There exists an operation $d$ corresponding to the exterior derivative such that

(a) the $d$ operation satisfies the Leibniz rule in the graded sense,

(b) $d^{2}=0$,

(c) the action on $A^{I}, \Psi$ and $C^{I}$ is defined as eq.(2.8).

3. The two operations are anticommuting: $d \delta_{B}+\delta_{B} d=0$.

4. There exists a *-conjugation which is an inner involution of the algebra $\mathcal{A}_{B}$ and antimultiplicative in the graded sense and also satisfies
(a) $\delta_{B} \circ^{*}={ }^{*} \circ \delta_{B}$,
(b) $d \circ^{*}={ }^{*} \circ d$.

5. The operations $\delta_{B}$ and $d$ are covariant: For any element $\rho \in \mathcal{A}_{B}$ they satisfy

(a) $\Delta_{L}\left(\delta_{B} \rho\right)=\left(i d \otimes \delta_{B}\right) \Delta_{L}(\rho)$,

(b) $\Delta_{L}(d \rho)=(i d \otimes d) \Delta_{L}(\rho)$.

In the non-deformed case the number of the ghost fields is the dimension of the adjoint representation, i.e., 3 for $S U(2)$. It is known that the property of the ghosts under the BRST transformation is related with the invariant one-forms on the group. In the $q$-deformed case, the result of the bicovariant bimodule calculus implies that the number of the independent bases of the invariant one-forms is 4 for the calculus on $\operatorname{Fun}_{q}(S U(2))$. They include both the adjoint and singlet representation. Although the ghost fields are not required to be the bimodule over $F u n_{q}(S U(2))$, it turns out that when we consider the covariant commutation relation with the different type of fields such as the matter fields, the projection onto the adjoint components are not compatible with the commutation relations. Therefore, in the $q$-deformed BRST algebra, we introduce the four ghosts $C^{I}$ where the suffix $I$ runs $0,-, 3,+$.

For the convenience for the following discussion, first we give here the definition of the ghosts $C^{I}$ :

\section{Definition 2:}

In the q-deformed BRST algebra based on the bicovariant differential calculus on $F u n_{q}(S U(2))$, we define the ghost field as a comodule represented by a $2 \times 2$ matrix $C^{i}{ }_{j}$. The left-coaction on it is

$$
\Delta_{L}\left(C^{i}{ }_{j}\right)=M_{i^{\prime}}^{i} \kappa\left(M_{j}^{j^{\prime}}\right) \otimes C_{j^{\prime}}^{i^{\prime}} \quad, \quad,
$$

and under the $*$-conjugation it transforms like a hermitian matrix:

$$
\left(C^{i}{ }_{j}\right)^{*}=C^{j}{ }_{i}
$$


We also introduce the upper index object $C^{i j}$ for convenience by using the spinor metric $\epsilon^{i j}$ :

$$
C^{i j}=C_{k}^{i} \epsilon^{k j}
$$

with this basis the coaction becomes simply

$$
\Delta_{L}\left(C^{i j}\right)=M_{i^{\prime}}^{i} M_{j^{\prime}}^{j} \otimes C^{i^{\prime} j^{\prime}} .
$$

We can decompose the ghost fields into singlet and adjoint representation by using the $q$-Pauli matrix given in eq.(1.4) as

$$
C^{I}=\sigma_{i_{1} i_{2}}^{I} C^{i_{1} i_{2}}
$$

where $I=0,-, 3,+$. The singlet component $C^{0}$ is invariant under the left-coaction.

Note that like eq.(2.14), in the following we freely change the suffix $I, J, .$. with double suffix $\left\{i_{1} i_{2}\right\},\left\{j_{1} j_{2}\right\} \ldots$ by using the $\sigma$ matrix.

The ghosts are the anticommuting fields in the BRST formalism, and therefore, to define the algebra of the ghosts $C^{I}$, we also impose the $q$-deformed anticommutativity among the ghosts. For this purpose, we take the same definition for the product rule as the one of the $\wedge$ product in the bicovariant differential calculus.

Finally, in the non-deformed case, the BRST transformation of the ghosts has the same form as the Maurer-Cartan equation. Therefore for the $q$-deformed BRST algebra, we postulate that the BRST transformation of the ghost fields has the same form as the Maurer-Cartan equation of the bicovariant differential calculus (1.12) and (1.13).

Here we summarize the conditions on the $q$-deformed ghosts:

\section{Condition B :}

1. As a comodule, they have the same properties as the right invariant basis $\theta_{j}^{i}$ appearing in the bicovariant differential calculus and consist of both adjoint and singlet components.

2. They are $q$-anticommuting.

3. The BRST transformation $\delta_{B}$ of the ghosts has the same form as the Maurer-Cartan equation obtained by the bicovariant differential calculus.

$$
\begin{aligned}
& \delta_{B} C^{a}=\frac{-i g}{q^{2}+q^{-2}} f_{b c}^{a} C^{b} C^{c}, \\
& \delta_{B} C^{0}=0,
\end{aligned}
$$

where $g$ is an arbitrary non-zero real constant. The structure constant $f_{b c}^{a}$ for $F u n_{q}(S U(2))$ is given in eq.(1.14).

To define the $q$-deformed BRST transformation of the matter, we take the algebraic representation in eq.(2.6). Therefore, using the above ghost fields we define the $q$-deformed BRST transformation of the matter analogously to eq.(2.6) as

$$
\delta_{B} \Psi=C^{I}\left(\Psi * \chi_{I}\right)=C^{a}\left(\Psi * \chi_{a}\right)+C^{0}\left(\Psi * \chi_{0}\right),
$$


where $\chi_{I} \in \mathcal{U}_{q}(S U(2))$ is the one given in eq.(1.10). Although the last term does not have the corresponding term in the non-deformed case, it goes to zero in the limit $q \rightarrow 1$. The singlet component of the ghost is not desirable from the physical point of view. On the other hand, as we shall see it seems it is necessary to include it in order to put the algebra into a simple form. We come back to this point in the discussion.

Therefore, for the $q$-deformed BRST transformation of the matter we have the following condition:

\section{Condition $\mathrm{C}$ :}

The BRST transformation of the matter fields is defined by the q-analogue of the infinitesimal transformation with the ghost as the parameter:

$$
\delta_{B} \Psi=C^{I}\left(\Psi * \chi_{I}\right),
$$

where the $\chi_{I}$ are the functionals given in eq.(1.10).

Finally we require the existence of the covariant derivative which is represented by the derivative $d$ and the gauge fields $A^{I}$. The coupling of the gauge fields to the matter fields is determined naturally by the structure of the BRST transformation of the matter fields given in eq.(2.18). Therefore, our requirement concerning the covariant derivative is:

Condition D :

There exists a covariant derivative $\nabla$ which acts on the matter as

$$
\nabla \Psi=d \Psi+A^{I}\left(\Psi * \chi_{I}\right)
$$

where $A^{I}$ are the gauge fields which satisfies

$$
\left(A_{j}^{i}\right)^{*}=A_{i}^{j}
$$

The covariant derivative transforms with the same rule as the corresponding matter

$$
\delta_{B} \nabla \Psi=C^{I}\left(\nabla \Psi * \chi_{I}\right)
$$

Under the $*$-conjugation it has the property

$$
\nabla \circ{ }^{*}{ }^{*} \circ \nabla
$$

In the following sections, requiring the above conditions and the covariance, we define the comodule algebra $\mathcal{A}_{B}$. The main part of the construction is to define the commutation relations $\mathcal{I}$. The relation $\mathcal{I}$ is defined by the following requirement.

\section{Condition E :}

1. The covariance, i.e. if the relation $r=0$ then $\Delta_{L}(r)=0$.

2. The consistency of the relations among each others.

3. Invariance under *-conjugation. 


\section{The BRST Algebra}

In this section we give the commutation among the elements and the BRST transformation of the gauge fields to complete the definition of the BRST algebra. For convenience, we give all relations in the first part of this section. The proof of the consistency of these relations are collected in the remaining part of the section.

\subsection{Results}

The commutation relation of each type of fields among themselves can be defined by taking the $q$-antisymmetric ( $q$-symmetric) product to vanish if it is a bosonic(fermionic) field in the limit $q \rightarrow 1$.

The ghost fields are $q$-anticommuting by definition. The gauge fields are also $q$-anticommuting since they are spacetime one-forms in the limit of $q \rightarrow 1$. We define the $q$-anticommutation relation of these fields using the same formula used to define the $\wedge$ product in ref.[CSWW]:

$$
\begin{aligned}
& \left(\mathcal{P}_{S}, \mathcal{P}_{S}\right)_{K L}^{I J} C^{K} C^{L}=0, \\
& \left(\mathcal{P}_{A}, \mathcal{P}_{A}\right)_{K L}^{I J} C^{K} C^{L}=0, \\
& \left(\mathcal{P}_{S}, \mathcal{P}_{S}\right)_{K L}^{I J} A^{K} A^{L}=0, \\
& \left(\mathcal{P}_{A}, \mathcal{P}_{A}\right)_{K L}^{I J} A^{K} A^{L}=0 .
\end{aligned}
$$

For the notation $(\cdot, \cdot)$ for the pair of projectors see appendix B.

The other relations including the derivative of the fields have also to be defined. They must satisfy the consistency condition E. Furthermore, since the operation $d$ relates some of the relations, they are not all independent. The independent relations are the ones between $\left(\left\{C^{I}\right\},\left\{d C^{I}\right\}\right),\left(\left\{A^{I}\right\},\left\{d A^{I}\right\}\right)$, $\left(\left\{C^{I}\right\},\left\{A^{I}\right\}\right),\left(\left\{C^{I}\right\},\{\Psi\}\right),\left(\left\{A^{I}\right\},\{\Psi\}\right),(\{\Psi\},\{\Psi\})$ and $(\{\Psi\},\{d \Psi\})$. The other relations can be derived from them by requiring the consistency with the $d$ operation.

When we require the consistency with other structures, we can also fix those relations. The resulting relations except the $(\{\Psi\},\{\Psi\})$ and the $(\{\Psi\},\{d \Psi\})$ relations are given by the following:

Proposition 1:

Define the ordering of the fields as

$$
\{\Psi, d \Psi\}>\left\{d A^{I}\right\}>\left\{A^{I}\right\}>\left\{d C^{I}\right\}>\left\{C^{I}\right\}
$$

then if $X>Y^{I}$, the commutation relation is given by

$$
X Y^{I}= \pm Y^{J}\left(X * \mathbf{L}_{J}^{I}\right)
$$

where the sign is taken as $+(-)$ if they are commuting(anticommuting) in the limit $q \rightarrow 1$ and $\mathbf{L}_{J}^{I}$ is the functional defined in eq.1.3). Note that we take the 1-form and the ghost anticommuting with each other.

The relations of $d A^{I}$ and $d C^{I}$ are

$$
\begin{aligned}
& \left(\mathcal{P}_{S}, \mathcal{P}_{A}\right)_{K L}^{I J} d A^{K} d A^{L}=0 \\
& \left(\mathcal{P}_{A}, \mathcal{P}_{S}\right)_{K L}^{I J} d A^{K} d A^{L}=0
\end{aligned}
$$


and

$$
\begin{aligned}
& \left(\mathcal{P}_{S}, \mathcal{P}_{A}\right)_{K L}^{I J} d C^{K} d C^{L}=0, \\
& \left(\mathcal{P}_{A}, \mathcal{P}_{S}\right)_{K L}^{I J} d C^{K} d C^{L}=0,
\end{aligned}
$$

The last two relations simply mean that $d A^{I}$ and $d C^{I}$ are $q$-commuting fields as expected.

The algebra of the matter fields can be defined like a quantum plane, since the quantum plane algebra is the algebra generated by the comodule imposing an appropriate commutation relation [Manin]. The algebra depends on the representation of the matter fields in the model. In our construction, we do not need to specify the representations of the matter. The algebra of the ghost and gauge fields which is defined in this section is applicable for any representation of the matter. This property provides the flexibility to consider the model with various matter fields. We give one example in the appendix C.

With the above relations we can find the BRST transformation of the gauge field by using the standard logic to define it in the field theory.

Proposition 2:

The BRST transformation of the gauge field is given by

$$
\begin{aligned}
& \delta_{B} A^{0}=d C^{0}, \\
& \delta_{B} A^{a}=d C^{a}-i g\left(\omega C^{0} A^{a}+f_{b c}^{a} C^{b} A^{c}\right),
\end{aligned}
$$

and it is nilpotent.

This completes the definition of the algebra. In the rest of this section we give the proof of the consistency of the above relations and the nilpotency of the BRST operation.

In the last part of this section we also define the field strength using the above algebra. The result is

Proposition 3:

The field strength is given by

$$
\begin{aligned}
& F^{a}=d A^{a}-\frac{i g}{q^{2}+q^{-2}} f_{b c}^{a} A^{b} A^{c} \\
& F^{0}=d A^{0}
\end{aligned}
$$

The field strength is covariant under the BRST transformation:

$$
\delta_{B} F^{I}=C^{J}\left(F^{I} * \chi_{J}\right),
$$

and satisfies the Bianchi identity:

$$
\begin{aligned}
d F^{a} & =\frac{i g}{q^{2}+q^{-2}} f_{b c}^{a}\left[A^{b} F^{c}-F^{b} A^{c}\right], \\
d F^{0} & =0 .
\end{aligned}
$$




\subsection{Algebra of Ghosts}

Here we prove the consistency of the commutation relation of the ghosts eq.(3.1) and eq.(3.2).

From the definitions (3.1) and (3.2), we obtain for the component of the adjoint representation:

$$
\begin{aligned}
C^{+} C^{+} & =0, \\
C^{-} C^{-} & =0, \\
C^{3} C^{3} & =\omega C^{-} C^{+}, \\
C^{+} C^{3} & =-q^{2} C^{3} C^{+}, \\
C^{-} C^{3} & =-q^{-2} C^{3} C^{-}, \\
C^{+} C^{-} & =-C^{-} C^{+} .
\end{aligned}
$$

The relations including the singlet component are

$$
\left[C^{0}, C^{a}\right]_{+}=\frac{-\omega}{q^{2}+q^{-2}} f_{b c}^{a} C^{b} C^{c}
$$

or explicitly,

$$
\begin{aligned}
& {\left[C^{+}, C^{0}\right]_{+}=q \omega C^{3} C^{+},} \\
& {\left[C^{-}, C^{0}\right]_{+}=q \omega C^{-} C^{3},} \\
& {\left[C^{3}, C^{0}\right]_{+}=\omega C^{+} C^{-} .}
\end{aligned}
$$

Finally we have

$$
C^{0} C^{0}=0
$$

Since the commutation relations among the ghosts are the same as the right invariant basis of the bicovariant bimodules, the conditions $\mathbf{B} 1$ and $\mathbf{B} 2$ are satisfied.

We also require that the BRST transformation is given by eqs.(2.15) and (2.16). As in the case of the bicovariant differential calculus, the commutation relation (3.25) implies that we can write eqs. 2.15) and (2.16) as the commutator with $C^{0}$ :

Proposition 4:

$$
\delta_{B} C^{I}=\frac{i g}{\omega}\left[C^{0}, C^{I}\right]_{+}
$$

It is important that the BRST transformation can be represented by the commutator, since it guarantees the Leibniz rule for the BRST operator (Cond.A 1a). The nilpotency of the BRST transformation (Cond.A1b) also holds on the algebra of the ghosts due to eq.(3.29).

The covariance of the $\delta_{B}$ (Cond.A5a) is also clear since $C^{0}$ is the invariant element and thus

$$
\Delta_{L}\left(\delta_{B} \rho\right)=\frac{1}{\omega} \Delta_{L}\left(\left[C^{0}, \rho\right]\right)=\left(i d \otimes \delta_{B}\right) \Delta_{L}(\rho) .
$$


In order to impose the hermiticity of the ghost field, the commutation relation (2.11) must be consistent with the algebra of the ghost fields defined above. This means that the $*$-operation which is antimultiplicative in the graded sense, i.e.,

$$
\left(C^{I} C^{J}\right)^{*}=-\left(C^{J}\right)^{*}\left(C^{I}\right)^{*}
$$

is a covariant inner involution of the algebra. This property is also clear since the algebra of the ghost is the same as the algebra of the right invariant basis $\theta^{I}$. In the present case, it is straightforward to prove these properties explicitly:

From the definition in eqs.(2.11) and (2.14), we find

$$
\begin{aligned}
& \left(C^{-}\right)^{*}=q C^{+}, \\
& \left(C^{+}\right)^{*}=q^{-1} C^{-}, \\
& \left(C^{3}\right)^{*}=C^{3}, \\
& \left(C^{0}\right)^{*}=C^{0} .
\end{aligned}
$$

By using these relation we see easily that the relations (3.19)-(3.29) are invariant under the *conjugation. The last equation together with eq.(3.30) implies also the condition A4a.

\subsection{Algebra of Matter and Ghost Field}

Next we add the matter fields consistently to the above algebra of the ghost fields. The commutation relation among the ghost and matter fields can be derived as follows:

First of all, the requirement of the covariance implies that the commutation relation is written in terms of linear combinations of the possible $\widehat{\mathbf{R}}$ matrix. Its consistency with the ghost algebra as well as the matter algebra implies that the matrix giving the commutation relation among the ghost and matter fields must satisfy the Yang-Baxter like equations and it excludes the linear combination of the $\widehat{\mathbf{R}}$ matrix like in the case of the other comodule algebra (see for example ref.[CSW]).

Therefore, for example if the matter $\Psi$ is bosonic and of the fundamental representation $\Psi^{i}$ as given in the appendix $\mathrm{C}$, then we can take the ansatz:

$$
\Psi^{k} C^{i_{1} i_{2}}=-\alpha \widehat{\mathbf{R}}_{j_{1} s}^{ \pm k i_{1}} \widehat{\mathbf{R}}_{j_{2} l}^{ \pm s i_{2}} C^{j_{1} j_{2}} \Psi^{l}
$$

where $\alpha$ is a constant which is 1 in the limit $q \rightarrow 1$. The \pm attached to the $\widehat{\mathbf{R}}$ matrix means that any combination is allowed and thus we have four possibilities. For the general representation we can write the ansatz as follows:

$$
\Psi C^{i_{1} i_{2}}= \pm \alpha C^{j_{1} j_{2}}\left(\Psi *\left(L_{\sigma j_{2}}^{i_{2}} * L_{\sigma^{\prime} j_{1}}^{i_{1}}\right) \circ \kappa\right)
$$

where we take the $+\operatorname{sign}$ for the bosonic and the - for the fermionic matter. The suffices $\sigma, \sigma^{\prime}= \pm$, and $L_{\sigma j}^{i}$ is the corresponding functional defined in eq.(A.4). It is easy to see that the relation (3.38) gives the commutation relation (3.37) for the boson of the fundamental representation $\Psi^{i}$. Using this ansatz, we can prove the following statement.

Proposition 5: 
The commutation relation among the ghost and matter is given by

$$
\Psi C^{I}= \pm C^{J}\left(\Psi * \mathbf{L}_{J}^{I}\right),
$$

where the $+(-)$ sign is taken for the bosonic(fermionic) matter.

Proof : First we impose the consistency with $*$-conjugation (Cond.E3). The hermiticity of $C_{j}^{i}$ in eq. (2.11) can be rewritten using the convention (2.12) as

$$
\left(C^{i j}\right)^{*}=C^{k l} \epsilon_{k j} \epsilon_{l i}
$$

Then the $*$-conjugation of the above ansatz $(3.38)$ with hermiticity 3.40$)$ gives the commutation relation between the $\Psi^{*}$ and $C^{i j}$ as

$$
\begin{aligned}
\left(C^{k_{1} k_{2}}\right) \Psi^{*} & = \pm \alpha^{*}\left(\Psi^{*} * \epsilon^{i_{2} k_{1}} \epsilon^{i_{1} k_{2}}\left(\Psi *\left(L_{\sigma j_{2}}^{i_{2}} * L_{\sigma^{\prime} j_{1}}^{i_{1}}\right) \circ \kappa\right)^{*} \circ \kappa \epsilon_{l_{1} j_{2}} \epsilon_{l_{2} j_{1}}\right) C^{l_{1} l_{2}}, \\
& = \pm \alpha^{*}\left(\Psi^{*} * \epsilon^{i_{2} k_{1}} \epsilon^{i_{1} k_{2}}\left(\kappa^{-1}\left(L_{\bar{\sigma}^{\prime} i_{1}}^{j_{1}}\right) * \kappa^{-1}\left(L_{\bar{\sigma} i_{2}}^{j_{2}}\right)\right) \epsilon_{l_{1} j_{2}} \epsilon_{l_{2} j_{1}}\right) C^{l_{1} l_{2}}, \\
& = \pm \alpha^{*}\left(\Psi^{*} * L_{\bar{\sigma}^{\prime} l_{2}}^{k_{2}} * L_{\bar{\sigma} l_{1}}^{k_{1}}\right) C^{l_{1} l_{2}},
\end{aligned}
$$

where if $\sigma= \pm$ then $\bar{\sigma}=\mp$ and the same rule for $\bar{\sigma}^{\prime}$.

The above equation is equivalent to

$$
\Psi^{*} C^{i_{1} i_{2}}= \pm \alpha^{*-1} C^{j_{1} j_{2}}\left(\Psi *\left(L_{\bar{\sigma}^{\prime} j_{2}}^{i_{2}} * L_{\bar{\sigma} j_{1}}^{i_{1}}\right) \circ \kappa\right) .
$$

The condition E3 implies that eq.(3.42) is equivalent to the relation (3.38) for the matter $\Psi^{*}$. Thus we get that

$$
L_{\sigma^{\prime} j}^{i}=L_{\bar{\sigma} j}^{i} \quad \text { and } \quad \alpha \alpha^{*}=1
$$

To fix the choice of the $\sigma$ we check the consistency with the BRST transformation. For the BRST transformation of the matter we use the form given in eq.(2.18) of Cond.C. For this part of the proof let us write the index of the matter field explicitly as $\Psi^{a}$ where $a$ runs over the corresponding representation. Taking the BRST transform of both sides of the relation, we get

$$
\begin{gathered}
\delta_{B}(\text { l.h.s. })=\frac{i g}{\omega}\left\{C^{0} \Psi^{a} C^{i_{1} i_{2}}-C^{J}\left(\Psi^{a} * \mathbf{L}_{J}^{0}\right) C^{i_{1} i_{2}} \pm \Psi^{a} C^{0} C^{i_{1} i_{2}} \pm \Psi^{a} C^{i_{1} i_{2}} C^{0}\right\}, \\
\delta_{B}(\text { r.h.s. })= \pm \alpha \frac{i g}{\omega}\left\{C^{0} C^{j_{1} j_{2}}\left(\Psi^{a} * \mathbf{L}_{\sigma j_{1} j_{2}}^{i_{1} i_{2}}\right)+C^{j_{1} j_{2}} C^{J}\left(\Psi^{a} * \mathbf{L}_{\sigma j_{1} j_{2}}^{i_{1} i_{2}} * \mathbf{L}_{J}^{0}\right)\right\},
\end{gathered}
$$

where $\mathbf{L}_{\sigma j_{1} j_{2}}^{i_{1} i_{2}}=\left(L_{\sigma j_{2}}^{i_{2}} * L_{\bar{\sigma} j_{1}}^{i_{1}}\right) \circ \kappa$.

Using the ansatz (3.38) again for the $\delta_{B}$ (l.h.s.), we can see the first terms of eqs.(3.44) and (3.45) are equivalent and thus we get the condition from the equivalence of eqs.(3.44) and (3.45):

$$
C^{J} C^{K}\left[\left(\Psi^{a} * \mathbf{L}_{J}^{0} * \mathbf{L}_{\sigma K}^{I}\right)-\alpha\left(\Psi^{a} * \mathbf{L}_{\sigma J}^{0} * \mathbf{L}_{\sigma K}^{I}\right)-\alpha\left(\Psi^{a} * \mathbf{L}_{\sigma J}^{I} * \mathbf{L}_{\sigma K}^{0}\right)\right]=-C^{J} C^{K}\left(\Psi^{a} * \mathbf{L}_{\sigma J}^{I} * \mathbf{L}_{K}^{0}\right)
$$

Multiplying the matrix $\widehat{\mathbf{R}}_{a I}^{L b}$

$$
\widehat{\mathbf{R}}_{a I}^{L b}=\widehat{\mathbf{R}}_{a i_{1}}^{\bar{\sigma} l_{1} a^{\prime}} \widehat{\mathbf{R}}_{a^{\prime} i_{2}}^{\sigma l_{2} b}
$$

where $\widehat{\mathbf{R}}_{b j}^{\sigma i a}$ is the $\widehat{\mathbf{R}}$ matrix of the fundamental representation (suffix $\mathrm{i}, \mathrm{j}$ ) and the representation of the matter field (suffix a,b). Taking the summation over $a$ and $I$ we get

$$
C^{K}\left(C^{I} * \mathbf{L}_{\bar{\sigma} K}^{J}\right)\left[\left(\Psi^{a} * \mathbf{L}_{J}^{0}\right)-\alpha\left(\Psi^{a} * \mathbf{L}_{\sigma J}^{0}\right)\right]=C^{I} C^{K}\left[\alpha\left(\Psi^{a} * \mathbf{L}_{\sigma K}^{0}\right)-\left(\Psi^{a} * \mathbf{L}_{K}^{0}\right)\right] .
$$


Since $\left(\Psi * \mathbf{L}_{+J}^{0}\right)$ and $\left(\Psi * \mathbf{L}_{-J}^{0}\right)=\left(\Psi * \mathbf{L}_{J}^{0}\right)$ terms are independent, we conclude that $\sigma=-$, i.e.,

$$
\mathbf{L}_{\sigma J}^{I}=\mathbf{L}_{-J}^{I}=\mathbf{L}_{J}^{I}
$$

and

$$
\alpha=1
$$

q.e.d.

We can deduce the following commutation relations immediately

Corollary 1 :

$$
\begin{aligned}
d \Psi C^{I} & =\mp C^{J}\left(d \Psi * \mathbf{L}_{J}^{I}\right) \\
\Psi d C^{I} & =d C^{J}\left(\Psi * \mathbf{L}_{J}^{I}\right) \\
d \Psi d C^{I} & =d C^{J}\left(d \Psi * \mathbf{L}_{J}^{I}\right)
\end{aligned}
$$

Proof : Applying the derivative $d$ on eq.(3.39) and comparing the term proportional to $d \Psi$ and $d C^{I}$ we get eqs.(3.51) and (3.52). Then taking again the derivative of eq.(3.51) or eq.(3.52) we get eq.(3.53).

By using the definition of the operator $\chi_{I}$, the commutation relation in (3.39) implies that we can write the BRST transformation of the matter field in eq.(2.18) as the commutator:

Proposition 6:

$$
\delta_{B} \Psi=\frac{i g}{\omega}\left[C^{0}, \Psi\right]_{\mp} \quad,
$$

where we take the commutator $[\cdot, \cdot]_{-}$for the bosonic matter and the anticommutator for fermionic matter.

Proof :

$$
\frac{i g}{\omega}\left[C^{0}, \Psi\right]_{\mp}=\frac{i g}{\omega}\left(C^{0} \Psi \mp \Psi C^{0}\right)=\frac{i g}{\omega}\left(C^{0} \Psi-C^{J} \Psi * L_{J}^{0}\right)=\frac{i g}{\omega} C^{i j} \Psi *\left(\varepsilon \sigma_{i j}^{0}-\sigma_{k l}^{0} \mathbf{L}_{i j}^{k l} .\right.
$$

Using the definition of $\chi_{I}$ in eq.(1.10) we have eq.(3.54).

q.e.d.

Once the BRST transformation is written in the form of a commutator with $C^{0}$, the Leibniz rule (Cond. A1a) follows immediately. Conds.A4a, 5a are also straightforward. The proof of the nilpotency (Cond.A1b) is

$$
\delta_{B}^{2} \Psi=\delta_{B}\left(C^{0} \Psi-\Psi C^{0}\right)=-C^{0}\left[C^{0} \Psi-\Psi C^{0}\right]-\left[C^{0} \Psi-\Psi C^{0}\right] C^{0}=0 .
$$

We also show the consistency of eq. 3.39 ) with the commutation relation of the ghosts and of the matter (Cond.E2), although we required it when we derived the ansatz (3.38):

In general the commutation relation of the matter is given by multiplying the projection operator on the product of two matter fields. Thus it is sufficient to prove that any projection $\mathcal{P}_{i_{1} . . i_{p}}^{l_{1} . l_{p}}$ of a tensor 
$\Psi^{i_{1} \ldots i_{p}}$ commutes with the relation 3.39 . Since any covariant projection operator is defined by a combination of the $\widehat{\mathbf{R}}$ matrix, we have

$$
\begin{aligned}
\mathcal{P}_{i_{1} . . i_{p}}^{l_{1} . l_{p}} \Psi^{i_{1} . . i_{p}} C^{I} & = \pm \mathcal{P}_{i_{1} . . i_{p}}^{l_{1} . l_{p}} C^{J} \mathbf{L}_{J}^{I}\left(M_{j_{1}}^{i_{1}} \ldots M_{j_{p}}^{i_{p}}\right) \Psi^{j_{1} . . j_{p}} C^{I} \\
& = \pm C^{J} \mathbf{L}_{J}^{I}\left(M_{l_{1}}^{l_{1}} \ldots M_{j_{p}}^{i_{p}}\right) \mathcal{P}_{j_{1} . . j_{p}}^{i_{1} . i_{p}} \Psi^{j_{1} . . j_{p}} C^{I}
\end{aligned}
$$

This proves the consistency with the relation of the matter fields.

The consistency of eq.(3.39) with the relation of the ghost in eqs.(3.1) and (3.2) can be proven as follows:

$$
\Psi C^{I} C^{K}=C^{J} C^{L}\left(\Psi * \mathbf{L}_{J}^{I} * \mathbf{L}_{L}^{K}\right)
$$

Thus the sufficient condition is that

$$
\left(\mathcal{P}_{r}, \mathcal{P}_{r^{\prime}}\right)_{K L}^{I J} \mathbf{L}_{S}^{K} * \mathbf{L}_{T}^{L}=\mathbf{L}_{K}^{I} * \mathbf{L}_{L}^{J}\left(\mathcal{P}_{r}, \mathcal{P}_{r^{\prime}}\right)_{S T}^{K L}
$$

This is equivalent to the condition

$$
\left(\widehat{\mathbf{R}}^{s}, \widehat{\mathbf{R}}^{s^{\prime}}\right)_{K L}^{I J} \mathbf{L}_{S}^{K} * \mathbf{L}_{T}^{L}=\mathbf{L}_{K}^{I} * \mathbf{L}_{L}^{J}\left(\widehat{\mathbf{R}}^{s}, \widehat{\mathbf{R}}^{s^{\prime}}\right)_{S T}^{K L}
$$

for all combination of $s$ and $s^{\prime}$ where $s, s^{\prime}=+,-$, since each projector $\mathcal{P}_{r}$ can be represented by the linear combination of $\widehat{\mathbf{R}}^{ \pm}$. Using the relation among $L_{+}$and $L_{-}$and the explicit form of $\left(\widehat{\mathbf{R}}^{s}, \widehat{\mathbf{R}}^{s^{\prime}}\right)$ given in the appendix $\mathrm{B}$, it is easy to see that eq. (3.60) is satisfied.

Using the similar method we can also derive the commutation relation of $\left(\left\{d C^{I}\right\},\left\{C^{I}\right\}\right)$ :

\section{Proposition 7 :}

The commutation relation among the ghosts and their derivatives is given by

$$
d C^{I} C^{J}=C^{K}\left(d C^{I} * \mathbf{L}_{K}^{J}\right)
$$

Proof : The first half of the proof of the proposition 5 concerning the consistency with the $*$-operation is also applicable here and we can set the relation as

$$
d C^{I} C^{J}=\alpha C^{K}\left(d C^{I} * \mathbf{L}_{\sigma K}^{J}\right)
$$

where $\alpha^{*} \alpha=1$. The hermiticity of the $d C_{j}^{i}$ can be simply imposed and does not require new relations.

Then we check the consistency with the BRST transformation. Taking the derivative of eq. 3.30) we get

$$
\delta_{B} d C^{I}=\frac{-i g}{\omega}\left\{\left[d C^{0}, C^{I}\right]_{-}+\left[d C^{I}, C^{0}\right]_{-}\right\} .
$$

Using the $\widehat{\mathbf{R}}$ matrix representation of eq.(3.62) we can see that if $\sigma=-(\sigma=+)$ then $d C^{0}\left(C^{0}\right)$ is commuting with all elements of $\left\{C^{I}\right\}\left(\left\{d C^{I}\right\}\right.$, resp.). Thus we get

$$
\delta_{B} d C^{I}=\left\{\begin{array}{lll}
-\frac{i g}{\omega}\left[d C^{I}, C^{0}\right]_{-} & \text {for } & \sigma=- \\
-\frac{i g}{\omega}\left[d C^{0}, C^{I}\right]_{-} & \text {for } & \sigma=+
\end{array} .\right.
$$


Now we see that if $\sigma=-$ then the BRST transform of $d C^{I}$ is the same as the matter and thus the consistency follows from the proposition 5. On the other hand, if $\sigma=+$ we can show that it contradicts with the nilpotency of the BRST operation as follows. Taking the BRST transform of the r.h.s. of eq.(3.65) we get

$$
\delta_{B}(\text { r.h.s. })=d C^{0} \delta_{B} C^{I}-d C^{J}\left(\delta_{B} C^{I} * \mathbf{L}_{+J}^{I}\right)
$$

The above two terms do not cancel and thus $\delta_{B}{ }^{2}$ on $d C^{I}$ is not zero with the choice $\sigma=+$. Finally, to get the Leibniz rule of the BRST operation on the product of $d C^{I}$ we must set $\alpha=1$. Thus we get eq.(3.61).

q.e.d.

The consistency of the relation (3.61) with the algebra of the ghosts is as follows. We use the projector expansion of the matrix $\left(\widehat{\mathbf{R}^{-1}}, \widehat{\mathbf{R}}\right)_{K L}^{I J}$ in eq. $(\overline{B .2})$ of the appendix B. Acting with $\left(\mathcal{P}_{S}, \mathcal{P}_{S}\right)$ and $\left(\mathcal{P}_{A}, \mathcal{P}_{A}\right)$ on both sides of eq.(3.61) we get

$$
\begin{aligned}
& \left(\mathcal{P}_{S}, \mathcal{P}_{S}\right)_{K L}^{I J}\left(d C^{K} C^{L}-C^{K} d C^{L}\right)=0 \\
& \left(\mathcal{P}_{A}, \mathcal{P}_{A}\right)_{K L}^{I J}\left(d C^{K} C^{L}-C^{K} d C^{L}\right)=0
\end{aligned}
$$

These relations show the consistency of the relation (3.61) with the eqs.(3.1) and (3.2) since eqs.(3.67) and (3.68) are the derivatives of eqs.(3.1) and (3.2), respectively. Furthermore eqs.(3.9) and (3.10) follow immediately .

\section{Corollary 2:}

$d C^{I}$ are $q$-commuting and their commutation relation is defined by eqs.(3.9) and (3.10).

Proof : Taking the derivative of eq.(3.61) we get

$$
d C^{I} d C^{J}=\left(\widehat{\mathbf{R}}^{-}, \widehat{\mathbf{R}}\right)_{K L}^{I J} d C^{K} d C^{L}
$$

where we have evaluated the functional $\mathbf{L}_{J}^{I}$. Using the projector expansion (B.2) in the appendix B, we get the eqs. 3.9$)$ and (3.10).

q.e.d.

\subsection{Gauge Field}

The BRST transformation of the gauge field can be derived by the usual logic used in the nondeformed gauge theory. The derivative of the field is not covariant under the BRST transformation. Its transformation is

$$
\begin{aligned}
\delta_{B} d \Psi & =-d \delta_{B} \Psi \\
& =-d\left[C^{I}\left(\Psi * \chi_{I}\right)\right] \\
& =C^{I}\left(d \Psi * \chi_{I}\right)-\left(d C^{I}\right)\left(\Psi * \chi_{I}\right)
\end{aligned}
$$

We define the covariant derivative $\nabla$ by introducing the gauge field $A^{I}$ as eq.(2.19) and we require the covariance under the BRST transformation 2.21) which can be rewritten as

$$
\begin{aligned}
\delta_{B} \nabla \Psi & =C^{I}\left(\nabla \Psi * \chi_{I}\right) \\
& =C^{I}\left(d \Psi * \chi_{I}\right)+C^{I} A^{J}\left(\Psi * \chi_{I} * \chi_{J}\right) .
\end{aligned}
$$


On the other hand taking the BRST transformation of the r.h.s. of eq.(2.19) we get

$$
\begin{aligned}
\delta_{B} \nabla \Psi & =-d \delta_{B} \Psi+\left(\delta_{B} A^{I}\right)\left(\Psi * \chi_{I}\right)-A^{I} \delta_{B}\left(\Psi * \chi_{I}\right) \\
& =-\left(d C^{I}\right) \Psi * \chi_{I}+C^{I}\left(d \Psi * \chi_{I}\right)+\left(\delta_{B} A^{I}\right)\left(\Psi * \chi_{I}\right)-A^{I} C^{J}\left(\Psi * \chi_{I} * \chi_{J}\right) .
\end{aligned}
$$

The BRST transformation of the gauge field can be defined by requiring the equivalence of the eqs. 3.71) and (3.72). Thus we get

$$
\left(\delta_{B} A^{I}\right)\left(\Psi * \chi_{I}\right)=\left(d C^{I}\right)\left(\Psi * \chi_{I}\right)+\left(A^{I} C^{J}+C^{I} A^{J}\right)\left(\Psi * \chi_{I} * \chi_{J}\right)
$$

In order to separate the fields from the generators $\chi_{I}$ of the dual algebra we need to reduce the $\left(\chi_{I} * \chi_{J}\right)$ in the second term into a term linear in the generators $\chi_{I}$. In the usual non-deformed case, we use the commutator for this purpose. The corresponding relation in the $q$-deformed case is eq.(1.15). Therefore, in our case $A^{I} C^{J}+C^{I} A^{J}$ need to create the projector $\mathbf{P}_{A d}$ to apply eq.(1.15).

Using the previous result we can prove that

\section{Proposition 8:}

The commutation relation among the ghost and gauge fields is given by

$$
A^{I} C^{J}=-C^{K}\left(A^{I} * \mathbf{L}_{K}^{J}\right) .
$$

With the above relation we can separate the algebra of $\chi_{I}$ and the BRST transformation of the gauge fields. Then, the BRST transformation of the gauge fields is given by

$$
\begin{aligned}
& \delta_{B} A^{0}=d C^{0}, \\
& \delta_{B} A^{a}=d C^{a}-i g\left(\omega C^{0} A^{a}+f_{b c}^{a} C^{b} A^{c}\right) .
\end{aligned}
$$

Proof : We can apply the same argument of the proof of the proposition 5 concerning the consistency with the $*$-operation and we can set

$$
A^{I} C^{J}=-\alpha C^{K}\left(A^{I} * \mathbf{L}_{\sigma K}^{J}\right)
$$

To fix the choice of $\sigma$ we apply the BRST transformation of eq.(3.77). The hermiticity of $A^{I}$ can be imposed simply and does not require any new condition. We know from eq.(3.73) that

$$
\delta_{B} A^{I}=d C^{I}+\left\{\text { other terms independent of } d C^{I}\right\}
$$

Thus comparing the $d C^{I}$ dependent term of the BRST transform of eq.(3.77) and eq.(3.61) we conclude that the relation of the ghost and gauge fields is eq.(3.74).

Using the definition of the $\mathbf{L}$, the relation (3.74) in terms of the $\widehat{\mathbf{R}}$ matrix is

$$
A^{I} C^{J}=-\left(\widehat{\mathbf{R}}^{-1}, \widehat{\mathbf{R}}\right)_{K L}^{I J} C^{K} A^{L}
$$

Substituting this into eq.(3.73), we get

$$
\begin{aligned}
\delta_{B} A^{I}\left(\Psi * \chi_{I}\right) & =\left(d C^{I}\right)\left(\Psi * \chi_{I}\right)+C^{K} A^{L}\left((\mathbf{1}, \mathbf{1})_{K L}^{I J}-\left(\widehat{\mathbf{R}}^{-1}, \widehat{\mathbf{R}}\right)_{K L}^{I J}\right)\left(\Psi * \chi_{I} * \chi_{J}\right), \\
& =\left(d C^{0}\right)\left(\Psi * \chi_{0}\right)+\left[d C^{a}-i g\left(\omega C^{0} A^{a}+f_{b c}^{a} C^{b} A^{c}\right)\right]\left(\Psi * \chi_{a}\right),
\end{aligned}
$$


where we have used eq.(B.3) in the appendix B. Thus we get eqs.(3.75) and (3.76)

Then applying the derivative $d$ on eq.(3.74), we get

Corollary 3 :

$$
\begin{aligned}
d A^{I} C^{J} & =C^{K}\left(d A^{I} * \mathbf{L}_{K}^{J}\right) \\
A^{I} d C^{J} & =d C^{K}\left(A^{I} * \mathbf{L}_{K}^{J}\right) \\
d A^{I} d C^{J} & =d C^{K}\left(d A^{I} * \mathbf{L}_{K}^{J}\right)
\end{aligned}
$$

Proof : Applying $d$ on eq.(3.74) and comparing the terms proportional to $d A^{I}$ and $d C^{I}$ we get eqs.(3.81) and (3.82). Applying $d$ on eq.(3.82) we get eq.(3.83).

To prove the nilpotency of the BRST transformation, we show that the BRST transformation of the gauge field can be also written by using the commutator with $C^{0}$.

Proposition 9:

The BRST transformation of the gauge field can be expressed as

$$
\delta_{B} A^{I}=d C^{I}+\frac{i g}{\omega}\left[A^{I}, C^{0}\right]_{+}
$$

and the BRST operation is nilpotent.

Proof : Using the decomposition of the $\left(\widehat{\mathbf{R}}^{-}, \widehat{\mathbf{R}}\right)$ given in the appendix $\mathrm{B}$, the relation of $A^{I}$ and $C^{I}$ can be written in components as

$$
\begin{aligned}
& A^{0} C^{0}=-C^{0} A^{0}, \\
& A^{0} C^{a}=-C^{a} A^{0}, \\
& A^{a} C^{0}=-\left(\omega^{2}+1\right) C^{0} A^{a}-\omega f_{b c}^{a} C^{b} A^{c}, \\
& A^{a} C^{b}=-C^{a} A^{b}-f_{a^{\prime}}^{a b}\left(\omega C^{0} A^{a^{\prime}}+f_{c d}^{a^{\prime}} C^{c} A^{d}\right) .
\end{aligned}
$$

The third relation can be written as

$$
\frac{1}{\omega}\left[A^{a}, C^{0}\right]_{+}=-\left(\omega C^{0} A^{a}+f_{b c}^{a} C^{b} A^{c}\right) .
$$

Comparing the above eqs.(3.85) and (3.89) with eqs. 3.75$)$ and (3.76), we get the formula given in eq.(3.84).

The nilpotency of the BRST operation on $A^{0}$ is apparent. The BRST operation on the gauge field $A^{a}$ can be proven as

$$
\begin{aligned}
\delta_{B}\left(\delta_{B} A^{a}\right) & =-d \delta_{B} C^{a}+\frac{i g}{\omega}\left[\delta_{B} A^{a}, C^{0}\right]_{-}, \\
& =\frac{i g}{\omega}\left(d\left[C^{0}, C^{a}\right]_{+}-\left[d C^{a}, C^{0}\right]_{-}+\frac{i g}{\omega}\left[\left[A^{a}, C^{0}\right]_{+}, C^{0}\right]_{-}\right) \\
& =-\frac{i g}{\omega}\left(\left[d C^{0}, C^{a}\right]_{-}\right)=0 .
\end{aligned}
$$


q.e.d.

Now using the BRST transformation of the gauge field given in the proposition 9 it follows:

Proposition 10 :

$$
\Psi A^{I}= \pm A^{I}\left(\Psi * \mathbf{L}_{J}^{I}\right)
$$

where we take the + sign for bosons and the - for fermions.

Proof : Applying again the same argument concerning the $*$-operation in the proposition 5 replacing $C^{I}$ with $A^{I}$, since $A^{I}$ is also given by a hermitian matrix, we can set the ansatz as:

$$
\Psi A^{I}= \pm \alpha A^{J}\left(\Psi * \mathbf{L}_{\sigma J}^{I}\right)
$$

To fix the choice of $\sigma$ we apply the BRST operation on eq.(3.92). Comparing the term proportional to $d C^{I}$ of the result with eq.(3.52), we conclude that $\sigma=-$ and $\alpha=1$.

q.e.d.

Corollary 4:

$$
\begin{aligned}
d \Psi A^{I} & =\mp A^{I}\left(d \Psi * \mathbf{L}_{J}^{I}\right) \\
\Psi d A^{I} & =d A^{I}\left(\Psi * \mathbf{L}_{J}^{I}\right) \\
d \Psi d A^{I} & =d A^{I}\left(d \Psi * \mathbf{L}_{J}^{I}\right)
\end{aligned}
$$

Proof : Apply the derivative $d$ on eq.(3.92), then eqs.(3.93) and (3.94) follow using the independence of the terms proportional to $d A^{I}$ and $d \Psi$. Applying again the derivative on eq.(3.93) we get eq.(3.95).

As a result of proposition 10, we can also prove the following relations

Corollary 5:

$$
\nabla \Psi=d \Psi+\frac{i g}{\omega}\left[A^{0}, \Psi\right]_{\mp}
$$

Proof : For the derivative part it is trivial. For the commutator part, replace the $C^{0}$ in eq.(3.55) with $A^{0}$ then we see that the second term in eq.(3.96) is equivalent to $A^{I}\left(\Psi * \chi_{I}\right)$.

From this representation of the covariant derivative, the Leibniz rule and the conditions eq.(2.22) follow immediately since $A^{0 *}=A^{0}$.

From the above results concerning the covariant derivative we can prove the following relation.

\section{Proposition 11:}

The BRST transformation of the covariant derivative of the matter is written as the commutator with $C^{0}$ :

$$
\delta_{B} \nabla \Psi=\frac{i g}{\omega}\left[C^{0}, \nabla \Psi\right]_{ \pm}
$$


and thus the nilpotency of the $\delta_{B}$ operator on $\nabla \Psi$ follows.

Proof : From the representation of the covariant derivative given in eq.(3.96), it is easy to show the relation

$$
\nabla \Psi C^{I}=\mp C^{J}\left(\nabla \Psi * \mathbf{L}_{J}^{I}\right) .
$$

We show that each term in eq.(3.98) satisfies the above relation separately. For the first term of eq.(3.96), eq.(3.98) is clear from eq.(3.51). Concerning the second term, due to eq.(3.85) $A^{0}$ simply makes the term opposite statistics and thus the commutation relation is followd from eq.(3.39) with the opposite sign. Thus, we get eq.(3.98). Then, replace the $\Psi$ with $\nabla \Psi$ and use the eq.(3.98) instead of eq. 3.39) in the proof of the proposition 6 .

q.e.d.

\subsection{Field Strength}

The field strength $F^{I}$ is defined by the square of the covariant derivative. When we apply it on the matter $\Psi$ we get

$$
\begin{aligned}
F^{I}\left(\Psi * \chi_{I}\right) & \equiv \nabla(\nabla \Psi) \\
& =d(\nabla \Psi)+A^{I}\left((\nabla \Psi) * \chi_{I}\right), \\
& =\left(d A^{I}\right)\left(\Psi * \chi_{I}\right)-\frac{1}{q^{2}+q^{-2}} A^{b} A^{c} f_{b c}^{a}\left[\left(\Psi * \mathbf{p} * \chi_{a}\right)+\omega\left(\Psi * \chi_{0} * \chi_{a}\right)\right] .
\end{aligned}
$$

Therefore, using eq.(1.16) we get the field strength as eqs.(3.13) and (3.14) in proposition 3. Using the commutation relation of the gauge field it can also be written as

$$
F^{I}=d A^{I}+\frac{i g}{\omega}\left[A^{0}, A^{I}\right]_{+}
$$

Using the covariance of the $\nabla^{2} \Psi$, we can prove the covariance of the gauge fields and derive the following commutation relations.

Proposition 12:

$$
F^{I} C^{J}=C^{K}\left(F^{I} * \mathbf{L}_{K}^{J}\right)
$$

Proof : It is straightforward to prove it directly using eqs. 3.74), (3.81) and 3.100). We can also derive the relation as follows: Since $\nabla^{2} \Psi$ is covariant we can apply the proof of the proposition 5 and thus

$$
\left[F^{I}\left(\Psi * \chi_{I}\right)\right] C^{J}=C^{K}\left(\left[F^{I}\left(\Psi * \chi_{I}\right)\right] * \mathbf{L}_{K}^{J}\right)=C^{K}\left(F^{I} * \mathbf{L}_{K^{\prime}}^{J}\right)\left(\Psi * \chi_{I} * \mathbf{L}_{K}^{K^{\prime}}\right)
$$

where we have used the coproduct of the functional $\mathbf{L}_{J}^{I}$. On the other hand the r.h.s. can be written as

$$
\text { r.h.s. }=F^{I} C^{K}\left(\Psi * \chi_{I} * \mathbf{L}_{K}^{J}\right) .
$$

From the equivalence of these two equations we get eq.(3.101).

Proposition 13: 
The field strength defined in eq.(3.100) is transformed under the BRST transformation as eq.(3.15) in proposition 3 and equivalently by the commutator as

$$
\delta_{B} F^{I}=\frac{i g}{\omega}\left[C^{0}, F^{I}\right]_{-} .
$$

Proof : The BRST transformation of the field strength is

$$
\begin{aligned}
\delta_{B} F^{a} & =-d \delta_{B} A^{a}+\frac{i g}{\omega}\left(\left[\delta_{B} A^{0}, A^{a}\right]_{-}+\left[\delta_{B} A^{a}, A^{0}\right]_{-}\right) \\
& =\frac{i g}{\omega}\left(-d\left[A^{a}, C^{0}\right]_{+}+\left[d C^{0}, A^{a}\right]_{-}+\left[d C^{a}, A^{0}\right]_{-}+\frac{i g}{\omega}\left[\left[A^{a}, C^{0}\right]_{+}, A^{0}\right]_{-}\right) \\
& =\frac{i g}{\omega}\left(\left[C^{0}, F^{a}\right]_{-}+\left[d C^{a}, A^{0}\right]_{-}\right) .
\end{aligned}
$$

Using the commutation relation (3.82) we get eq.(3.104). Then using eq.(3.101), we get eq.(3.15). q.e.d.

\section{Proposition 14 :}

The commutation relation of the fields $A^{I}$ and their derivatives $d A^{I}$ is given by

$$
d A^{I} A^{J}=A^{K}\left(d A^{I} * \mathbf{L}_{K}^{J}\right)
$$

Proof : Using the same argument concerning the $*$-conjugation in the proof of the proposition 5, they must satisfy

$$
d A^{I} A^{J}=\alpha A^{K}\left(d A^{I} * \mathbf{L}_{\sigma K}^{J}\right)
$$

Take the BRST transformation of the eq.(3.107) and compare the term proportional to the derivative of the ghost $d C^{I}$. Then, we find

$$
d A^{I} d C^{J}=\alpha d C^{K}\left(d A^{I} * \mathbf{L}_{\sigma K}^{J}\right)
$$

Since this must be equivalent to eq.(3.83), we conclude $\alpha=1$ and $\sigma=-$. The consistency of the eq. 3.106) with the commutation relation among the $A^{I}$ can be shown analogously to the ghost case by replacing $C^{I}$ and $d C^{I}$ in eqs.(3.67) and (3.68).

q.e.d.

\section{Corollary 6 :}

$d A^{I}$ are $q$-commuting and their commutation relation is defined by eqs.(3.7) and (3.8).

Proof : Taking the derivative of eq.(3.106) and use the same formula used in the proof of the corollary 2.

q.e.d.

Finally we give the formula which is the simpler representation of the Bianchi identity in eq.(3.16) of proposition 3 . 


\section{Proposition 15:}

The field strength $F^{I}$ satisfies the following relation

$$
d F^{I}=\frac{i g}{\omega}\left[F^{I}, A^{0}\right]_{-} .
$$

Proof : Taking the derivative of the field strength $F^{I}$ given in eq.(3.100), we get

$$
d F^{I}=\frac{i g}{\omega}\left\{\left[d A^{0}, A^{I}\right]_{-}+\left[d A^{I}, A^{0}\right]_{-}\right\}=\frac{i g}{\omega}\left[d A^{I}, A^{0}\right]_{-} .
$$

On the other hand using the relation $A^{0} A^{0}=0$ we get

$$
\left[F^{I}, A^{0}\right]_{-}=\left[d A^{I}, A^{0}\right]_{-}+\frac{i g}{\omega}\left[\left[A^{0}, A^{I}\right]_{+}, A^{0}\right]_{-}=\left[d A^{I}, A^{0}\right]_{-} .
$$

Thus we get eq.(3.109).

q.e.d.

\subsection{Proof of Propositions 1-3 and Summary}

We complete here the proof of the propositions 1, 2, and 3:

Proof of proposition 1

All commuation relations among the fields given in the propositions 5, 7, 8, 10, 14 and the corollaries $1,3,4$, can be summarized as in eqs. (3.5) and (3.6). The corollary 2 and 6 prove (3.7)-(3.10).

q.e.d.

Proof of proposition 2

Substituting the explicit relations $(3.85)$ and (3.89) into the eq.(3.84) in the proposition 9, we get the eqs.(3.11) and (3.12). Then the nilpotency follows from the proposition 9.

q.e.d.

Proof of proposition 3

As we mentioned eq. 3.99) gives the definition of the field strength as eqs.(3.13) and (3.14). Using the commutation relation given in the proposition 12, the BRST transformation of $F^{I}$ given in proposition 13 can be written as eq.(3.15). The Bianchi identity in proposition 3 can be derived as follows: As for the $F^{0}$ it is trivial. To prove eq.(3.16), we substitute eq.(3.13) into the r.h.s of eq.(3.109). Since the commutation relation of $A^{I}$ and $C^{I}$ are given by the same formula (3.1)-(3.4), we have the relation for the $\left[A^{0}, A^{a}\right]_{+}$corresponding to eq.(3.25). Therefore we get

$$
\begin{aligned}
{\left[F^{a}, A^{0}\right]_{-} } & =\frac{-\omega}{q^{2}+q^{-2}} f_{b c}^{a} d\left(A^{b} A^{c}\right)-\frac{i g}{q^{2}+q^{-2}} f_{b c}^{a}\left[A^{b} A^{c}, A^{0}\right] \\
& =\frac{-\omega}{q^{2}+q^{-2}}\left\{f_{b c}^{a}\left(d A^{b} A^{c}-A^{b} d A^{c}\right)+\frac{i g}{\omega} f_{b c}^{a}\left(-\left[A^{b}, A^{0}\right] A^{c}+A^{b}\left[A^{c}, A^{0}\right]\right)\right\}
\end{aligned}
$$

Using eq.(3.100), we get eq.(3.16). 
The propositions 4, 6, 11, 13 can be summarized as: the BRST transformation of the fields except gauge fields can be represented by the commutator with $C^{0}$. Concerning the gauge field, the proposition 9 shows that the homogeneous term of the BRST transformation is again a commutator with $C^{0}$.

The above property is very important in the present construction of the BRST algebra, since due to this property, the Leibniz rule of the operator $\delta_{B}$ is satisfied in rather trivial way. The nilpotency becomes also apparent since $\left(C^{0}\right)^{2}=0$.

\section{Discussion}

We have constructed the $q$-deformed BRST algebra which corresponds to the $q$ deformation of the algebra of the ghost, gauge and matter fields on one spacetime point. To obtain the $q$-deformation of the BRST formulation of the gauge field theory, we have to take the structure of the base manifold into consideration. Using the result here, one may take the base manifold as a usual spacetime but a more interesting possibility is the one when the base manifold is also described by the non-commutative function algebra. In both cases, we have to reconsider the meaning of the usual quantization so that it fit to the pure algebraic formulation.

In the latter case, we also have to consider the new theory of the gravity based on the noncommutative geometry. Then we can ask the interesting question whether the new deformation parameter may play the role of the cut off of the quantum gravity. Our result here may also shed some light on that direction, since the local Lorentz symmetry can be treated as usual gauge symmetry. It is known that there is a quantum deformation of the Lorentz group [CSSW,PW] and thus one may write down the $q$-deformed BRST algebra using the result of the differential calculus on the quantum Lorentz group [CDSWZ,CW,SWW].

In the construction presented here, we used the singlet component in the hermitian matrix. Especially the proof of the nilpotency and Leibniz rules became simple, since all action of the $\delta_{B}$ operator is represented by the commutator with $C^{0}$. Nevertheless, it is very interesting if there is a $q$-deformed BRST algebra without singlet component.

Concerning the ghost algebra, it is possible to obtain the algebra without the singlet component. First of all, the Maurer-Cartan equation does not contain the singlet component and thus the BRST transformation of the ghosts does not either. The algebra of $\chi_{I}$ contains the singlet component but it appears in the functional $\mathbf{p}$ and this $\mathbf{p}$ is central in the algebra, therefore we can divide it out. Then we get the algebra where the commutation relations are given by setting $\mathbf{p}=i$ in the eqs.(1.18)-(1.20). With this new operator $\tilde{\chi}_{a}=\chi_{a} * \mathbf{p}^{-1}$ we can write $\delta_{B} \Psi=C^{a}\left(\Psi * \tilde{\chi}_{a}\right)$ and prove that $\delta_{B}{ }^{2}=0$. In this way we can remove the singlet component in the BRST transformation and thus we do not need the singlet component of the gauge field. The trouble occurs when we start to consider the commutation relations among the different types of fields such as gauge and ghost fields without the singlet component. We can not use the $\widehat{\mathbf{R}}$ matrix which is defined in the standard way for the tensor representation. For example if we simply take

$$
C^{a} A^{b}=\widehat{\mathbf{R}}_{c d}^{a b} A^{c} C^{d}
$$

where $\widehat{\mathbf{R}}_{c d}^{a b}$ is the $\widehat{\mathbf{R}}$ matrix of adjoint representation. If we take the $*$-conjugation and using the hermiticity of the fields,

$$
A^{a} C^{b}=\widehat{\mathbf{R}}_{c d}^{a b} A^{c} C^{d}
$$


and thus we can not impose the hermiticity condition.

\section{Acknowledgement}

The author would lilke to acknowledge U. Carow-Watamura, M. Hirayama, P.P. Kulish, R. Sasaki for interesting and critical discussions. He thanks K. Ueno and T. Masuda for helpful discussions on the mathematical aspects. He would also like to acknowledge M. Schmidt for his kind hospitality during the stay at the Institute for Theoretical Physics of the Heidelberg University.

\section{Appendix}

\section{A Functionals and Convolution Product}

The quantum matrix is denoted by $M_{j}^{i}$ and quantum group relations are defined by

$$
\begin{aligned}
\widehat{\mathbf{R}}_{k^{\prime} l^{\prime}}^{i j} M_{k}^{k^{\prime}} M_{l}^{l^{\prime}} & =M_{i^{\prime}}^{i} M_{j^{\prime}}^{j} \widehat{\mathbf{R}}_{k l}^{i^{\prime} j^{\prime}}, \\
\epsilon_{i j} M_{k}^{i} M_{l}^{j} & =\epsilon_{k l}, \\
\left(M_{j}^{i}\right)^{*} & =\kappa\left(M_{i}^{j}\right) .
\end{aligned}
$$

There are functionals defined by the following relations

$$
L_{ \pm j}^{i}\left(M_{l}^{k}\right)=\widehat{\mathbf{R}}_{l j}^{ \pm i k}
$$

where the normalization of the $\widehat{\mathbf{R}}$ matrix is

$$
\widehat{\mathbf{R}}=q^{\frac{1}{2}} \mathcal{P}_{S}-q^{\frac{-3}{2}} \mathcal{P}_{A}
$$

They satisfy the following relations

$$
\begin{aligned}
\widehat{\mathbf{R}}_{k^{\prime} l^{\prime}}^{i j} L_{ \pm l}^{l^{\prime}} * L_{ \pm k}^{k^{\prime}} & =L_{ \pm j^{\prime}}^{j} * L_{ \pm i^{\prime}}^{i} \widehat{\mathbf{R}}_{k l}^{i^{\prime} j^{\prime}}, \\
\widehat{\mathbf{R}}_{k^{\prime} l^{\prime}}^{i j} L_{+l}^{l^{\prime}} * L_{-k}^{k^{\prime}} & =L_{-j^{\prime}}^{j} * L_{+i^{\prime}}^{i} \widehat{\mathbf{R}}_{k l}^{i^{\prime} j^{\prime}} \\
\left(L_{+j}^{i}\right)^{*} & =\kappa^{-1}\left(L_{-i}^{j}\right) .
\end{aligned}
$$

The convolution product of two functionals $f, g \in \mathcal{U}_{q}(s u(2))$ is defined by

$$
f * g=(f \otimes g) \Delta .
$$

We use the convolution product $*$ between a functional $f$ over the Hopf algebra, e.g. $f \in \mathcal{U}_{q}(s u(2))$ and a (left)comodule $\rho$ as (See also the last section of $[\mathrm{CW}]$.)

$$
(\rho * f) \equiv(f \otimes i d) \Delta_{L}(\rho)
$$

The $*$-operation is defined as

$$
(\rho * f)^{*}=\left(\rho^{*} * f^{*} \circ \kappa\right) .
$$

With these definitions we have the following associativity

$$
\rho *(f * g)=(\rho * f) * g
$$

due to the relation $(\Delta \otimes i d) \Delta_{L}=\left(i d \otimes \Delta_{L}\right) \Delta_{L}$. 


\section{B $\widehat{\mathbf{R}}$ matrix and Projectors}

Given tensors $A_{k l}^{i j}$ and $B_{k l}^{i j}$ we define

$$
(A, B)_{K L}^{I J}=(A, B)_{k_{1} k_{2} l_{1} l_{2}}^{i_{1} i_{2} j_{1} j_{2}}=\widehat{\mathbf{R}}_{j_{1}^{\prime} i_{2}^{\prime}}^{-i_{2} j_{1}} A_{k_{1} l_{1}^{\prime}}^{i_{1} j_{1}^{\prime}} B_{k_{2}^{\prime} l_{2}}^{i^{\prime} j_{2}} \widehat{\mathbf{R}}_{k_{2} l_{1}}^{l_{1}^{\prime} k_{2}^{\prime}}
$$

Applying this notation the pair of the $\widehat{\mathbf{R}}$ matrix, $\left(\widehat{\mathbf{R}}^{-1}, \widehat{\mathbf{R}}\right)$ can be expanded by the projection operators as

$$
\left(\widehat{\mathbf{R}}^{-1}, \widehat{\mathbf{R}}\right)_{K L}^{I J}=\left(\left(\mathcal{P}_{S}, \mathcal{P}_{S}\right)+\left(\mathcal{P}_{A}, \mathcal{P}_{A}\right)-q^{2}\left(\mathcal{P}_{A}, \mathcal{P}_{S}\right)-q^{-2}\left(\mathcal{P}_{S}, \mathcal{P}_{A}\right)\right)_{K L}^{I J}
$$

Note that each term $\left(\mathcal{P}_{r}, \mathcal{P}_{r^{\prime}}\right)$ is a projection operator. (see also ref.[CSWW].) Then using the $\sigma^{I}$ matrices we can prove the following relations.

$$
\begin{aligned}
& \delta_{k_{1}}^{i_{1}} \delta_{k_{2}}^{i_{2}} \delta_{l_{1}}^{j_{1}} \delta_{l_{2}}^{j_{2}}-\widehat{\mathbf{R}}_{k_{1} i_{3}}^{-i_{1} j_{3}} \widehat{\mathbf{R}}_{j_{3} i_{4}}^{-i_{2} j_{1}} \widehat{\mathbf{R}}_{j_{4} l_{2}}^{i_{4} j_{2}} \widehat{\mathbf{R}}_{k_{2} l_{1}}^{i_{3} j_{4}} \\
& =\sigma_{a}^{i_{1} i_{2}} \sigma_{k_{1} k_{2}}^{a} \sigma_{0}^{j_{1} j_{2}} \sigma_{l_{1} l_{2}}^{0}-\sigma_{0}^{i_{1} i_{2}} \sigma_{k_{1} k_{2}}^{a} \sigma_{a}^{j_{1} j_{2}} \sigma_{l_{1} l_{2}}^{0}+\sigma_{0}^{i_{1} i_{2}} \sigma_{k_{1} k_{2}}^{0} \sigma_{a}^{j_{1} j_{2}} \sigma_{l_{1} l_{2}}^{a}-\left(\omega^{2}+1\right) \sigma_{a}^{i_{1} i_{2}} \sigma_{k_{1} k_{2}}^{0} \sigma_{0}^{j_{1} j_{2}} \sigma_{l_{1} l_{2}}^{a} \\
& -\omega f_{a}^{b c} \sigma_{b}^{i_{1} i_{2}} \sigma_{k_{1} k_{2}}^{0} \sigma_{c}^{j_{1} j_{2}} \sigma_{l_{1} l_{2}}^{a}-\omega f_{b c}^{a} \sigma_{a}^{i_{1} i_{2}} \sigma_{k_{1} k_{2}}^{b} \sigma_{0}^{j_{1} j_{2}} \sigma_{l_{1} l_{2}}^{c}+\left(q^{2}+q^{-2}\right) \mathbf{P}_{A d c d}^{a b} \sigma_{a}^{i_{1} i_{2}} \sigma_{k_{1} k_{2}}^{c} \sigma_{b}^{j_{1} j_{2}} \sigma_{l_{1} l_{2}}^{d},
\end{aligned}
$$

and

$$
\begin{aligned}
\delta_{k_{1}}^{i_{1}} & \delta_{k_{2}}^{i_{2}} \delta_{l_{1}}^{j_{1}} \delta_{l_{2}}^{j_{2}}-\widehat{\mathbf{R}}_{k_{1} i_{3}}^{i_{1} j_{3}} \widehat{\mathbf{R}}_{{ }_{j_{3} i_{4}} i_{2} j_{1}} \widehat{\mathbf{R}}_{j_{4} l_{2}}^{-i_{4} j_{2}} \widehat{\mathbf{R}}_{k_{2} l_{1}}^{i_{3} j_{4}} \\
= & \sigma_{a}^{i_{1} i_{2}} \sigma_{k_{1} k_{2}}^{a} \sigma_{0}^{j_{1} j_{2}} \sigma_{l_{1} l_{2}}^{0}-\sigma_{a}^{i_{1} i_{2}} \sigma_{k_{1} k_{2}}^{0} \sigma_{0}^{j_{1} j_{2}} \sigma_{l_{1} l_{2}}^{a}+\sigma_{0}^{i_{1} i_{2}} \sigma_{k_{1} k_{2}}^{0} \sigma_{a}^{j_{1} j_{2}} \sigma_{l_{1} l_{2}}^{a}-\left(\omega^{2}+1\right) \sigma_{0}^{i_{1} i_{2}} \sigma_{k_{1} k_{2}}^{a} \sigma_{a}^{j_{1} j_{2}} \sigma_{l_{1} l_{2}}^{0} \\
& \quad-\omega f_{a}^{b c} \sigma_{b}^{i_{1} i_{2}} \sigma_{k_{1} k_{2}}^{a} \sigma_{c}^{j_{1} j_{2}} \sigma_{l_{1} l_{2}}^{0}-\omega f_{b c}^{a} \sigma_{0}^{i_{1} i_{2}} \sigma_{k_{1} k_{2}}^{b} \sigma_{a}^{j_{1} j_{2}} \sigma_{l_{1} l_{2}}^{c}+\left(q^{2}+q^{-2}\right) \mathbf{P}_{A d c d}^{a b} \sigma_{a}^{i_{1} i_{2}} \sigma_{k_{1} k_{2}}^{c} \sigma_{b}^{j_{1} j_{2}} \sigma_{l_{1} l_{2}}^{d},
\end{aligned}
$$

Thus the product of the two hermitian fields $A^{I}$ and $C^{J}$ can be expanded as

$$
A^{K} C^{L}\left((\mathbf{1}, \mathbf{1})-\left(\widehat{\mathbf{R}}, \widehat{\mathbf{R}}^{-}\right)\right)_{K L}^{I J}\left(\chi_{I} * \chi_{J}\right)=i g\left(-\omega A^{0} C^{a}-f_{b c}^{a} A^{b} C^{c}\right) \chi_{a}
$$

\section{Algebra of Matter field}

We give here an example of the algebra of matter fields. In general, once we define the representation of the matter fields, we construct the corresponding $q$-antisymmetrizer and $q$-symmetrizer. Then if one wants to define the fermions, we impose the $q$-symmetric product to vanish and the $q$-antisymmetric product to vanish for the bosons.

When the matter is bosonic and of the fundamental representation $\Psi^{i}$, the algebra is defined like the differential calculus on the quantum space [Pusz,WZ](see also [CSW].), and we can set the following commutation relations:

$$
\begin{aligned}
\mathcal{P}_{A k l}^{i j} \Psi^{k} \Psi^{l} & =0 \\
d \Psi^{i} \Psi^{j}-q^{\frac{3}{2}} \Psi^{k}\left(\Psi^{i} * L_{+k}^{j}\right) & =0
\end{aligned}
$$


and the conjugated relation for the fields $\Psi^{* i}$ and $d \Psi^{* i}$. The relation between the $\Psi^{i}$ and $\Psi^{* i}$ can be defined for example as

$$
\Psi^{* i} \Psi^{j}=\beta \Psi^{k}\left(\Psi^{* i} * L_{+k}^{j}\right),
$$

where $\beta$ is a constant which is 1 in the limit $q \rightarrow 1$. Then, by the consistency with derivative $d$, it is easy to show the following relations:

$$
\begin{aligned}
\Psi^{* i} d \Psi^{j} & =\beta d \Psi^{k}\left(\Psi^{* i} * L_{+k}^{j}\right), \\
d \Psi^{* i} d \Psi^{j} & =-\beta d \Psi^{k}\left(d \Psi^{* i} * L_{+k}^{j}\right), \\
\mathcal{P}_{S_{k l}}^{i j} d \Psi^{k} d \Psi^{l} & =0,
\end{aligned}
$$

and their $*$-conjugation.

\section{References}

[Abe] E. Abe, "Hopf Algebras", Cambridge Tracts in Math., vol.74, Cambridge Univ. Press, 1980.

[Are] I.Ya. Aref'eva, I.V. Volovich, Quantum group gauge fields. Mod. Phys. Lett. A6 (1991) 893-907.

[Ber] D. Bernard, Prog. Theor. Phys. Suppl. 102 49-66 (1990).

[BM] T. Brzeziński and S. Majid, "Quantum group gauge theory on quantum spaces." Preprint DAMTP/92-27, 1992; "Quantum Group Gauge Theory on Classical Spaces" Preprint DAMTP/92-51.

[BRST] C. Becchi, A. Rouet and R. Stora, Ann. Phys. 98 287-321 (1976);

I.V. Tyuitin, Lebedev preprint FIAN 39 (1975), unpublished.

[Carow] U. Carow-Watamura, Ph.D. Thesis, "The quantum group symmetry in the models of elementary particle physics."

[CDSWZ] C. Chryssomalakos, B. Drabant, M. Schlieker, W. Weich and B. Zumino, Commun. Math. Phys. 147, 635-646 (1992).

[Connes] A. Connes, Non-commutative differential geometry, Technical Report 62, IHES, 1986.

[CSSW] U. Carow-Watamura, M. Schlieker, M. Scholl and S. Watamura, Z.Phys. C - Particles and Fields 48, 159-165 (1990); Int.Jour. of Mod.Phys. A6, 3081-3108 (1991).

[CSW] U. Carow-Watamura, M. Schlieker and S. Watamura, Z.Phys. C - Particles and Fields 49, 439-446 (1991). 
[CSWW] U. Carow-Watamura, M. Schlieker, S. Watamura and W. Weich, Commun. Math. Physics 142, 605-641 (1991).

[CW] U. Carow-Watamura and S. Watamura, "Complex Quantum Group, Dual Algebra and Bicovariant Differential Calculus," preprint TU-382(1991) to be published in Commun. Math. Physics.

[Dri] V.G. Drinfeld, "Quantum Groups", Proceedings of the International Congress of Mathematicians, 1986, Vol.1, 798-820.

[FP] L.D. Faddeev and V. Popov, Phys. Lett. 25B, 29-30 (1967).

[FRT] L.D. Faddeev, N.Yu. Reshetikhin, and L.A. Takhtajan, Quantization of Lie groups and Lie algebras. Algebra i Analiz, 1 178-206 (1989).

[Hira] M. Hirayama, Gauge Field Theory of the Quantum Group $S U_{q}(2)$, preprint TOYAMA-74 1992.

[IP] A.P. Isaev and Z. Popowicz Phys. Lett. 281B(1992)271-278.

[Jim] M. Jimbo, Lett. Math. Phys. 10, 63-69 (1986).

[Jur] B. Jurco, Lett. Math. Phys. 22, 177-186 (1991).

[KO] T. Kugo and I. Ojima, Prog. Theore. Phys. Supple. 66 1-130 (1979).

[Manin] Yu. I. Manin, "Quantum groups and non-commutative geometry", Centre de Recherches Mathematiques, Universite de Montreal, (1988).

[Pusz] W. Pusz and S.L. Woronowicz, Reports Math. Phys. 27,231-257(1989).

[PW] P. Podlés and S.L. Woronowicz, Commun. Math. Phys. 130, 381-431 (1990).

[Rosso] M. Rosso, Duke Math. J. 61, 11-40 (1990).

[SWW] X. Sun, S. Wang and K. Wu, "Differential Calculus on Quantum Lorentz Group," preprint CCAST-92-14(1992).

[Weich] W. Weich, Ph.D. Thesis, "Die Quantengruppe $S U_{q}(2)$ - kovariante Differentialrechnung und ein quantensysmmetrisches quantenmechanisches Modell", Karlsruhe University, Norvember 1990.

[Wor1] S.L. Woronowicz, Commun. Math. Phys. 111, 613-665 (1987).

[Wor2] S.L. Woronowicz, Commun. Math. Phys. 122, 122-170 (1989).

[WuZ] K. Wu and R. Zhang, Commun. Theor. Phys. 17, 175-182 (1992).

[WZ] J. Wess and B. Zumino. Covariant differential calculus on the quantum hyperplane. Nucl. Phys. B (Proc. Suppl.) 18 B 302-312, (1990). 